Jõ̃o Bortoletti FILHO ${ }^{1}$

LUCIANO MARCONDES

MACHADO NARDOZZA ${ }^{2}$

EDWARD Araujo JÚNIOR ${ }^{3}$

LílIAM CRISTINe RÔlO ${ }^{4}$

PAULO MARTIN NOWAK ${ }^{1}$

ANTONIO FERNANDES MORON ${ }^{5}$

Artigos originais

Palavras-chave

Estruturas embrionárias Comprimento cabeça-cóccix Valores de referência

Primeiro trimestre da gravidez Imagem tridimensional

Keywords

Embryonic structures Crown-rump length Reference values

Pregnancy trimester first Imaging, three-dimensional

\section{Volume do embrião estimado pela ultra-sonografia tridimensional entre a sétima e a décima semana de gestação}

Embryo volume estimated by three-dimensional ultrasonography at seven to ten weeks of pregnancy

\section{Resumo}

OBJETIVO: avaliar a evolução do volume do embrião (VE) entre a sétima e a décima semana de gestação por meio da ultra-sonografia tridimensional. MÉTODOS: realizou-se um estudo de corte transversal com 63 gestantes normais entre a sétima e a décima semana. Os exames ultra-sonográficos foram realizados por meio de um transdutor endocavitário volumétrico. Para o cálculo do VE, utilizou-se o método VOCAL (Virtual Organ Computer-aided Analysis) com ângulo de rołação de $12^{\circ}$, com delimitação de 15 planos seqüenciais. Para o VE foram calculadas médias, medianas, desvios padrão e valores máximo e mínimo em todas as idades gestacionais. Para se avaliar a correlação entre o VE e o comprimento cabeça-nádega (CCN) foi criado gráfico de dispersão, sendo o ajuste realizado pelo coeficiente de determinação $\left(R^{2}\right)$. Para se determinarem intervalos de referência do VE em função do CCN, utilizou-se a seguinte fórmula: percentil =VE+K versus dp, com K=1,96. RESULTADOS: o CCN variou de 9,0 a 39,7 mm, com média de $23,9 \mathrm{~mm}( \pm 7,9 \mathrm{~mm})$, enquanto o VE variou de 0,1 a $7,6 \mathrm{~cm}^{3}$, com média de 2,7 $\mathrm{cm}^{3}\left( \pm 3,2 \mathrm{~cm}^{3}\right)$. O VE foi altamente correlacionado com o CCN, sendo que o melhor ajuste foi obtido com regressão quadrática $(V E=0,165-0,055$ $\left.\times C C N+0,005 \times C_{C N}^{2} ; R^{2}=0,853\right)$. O VE médio variou de $0,1\left(-0,3\right.$ a 0,5 $\left.\mathrm{cm}^{3}\right)$ a 6,7 $\mathrm{cm}^{3}\left(3,8\right.$ a $\left.9,7 \mathrm{~cm}^{3}\right)$ no intervalo de 9 a $40 \mathrm{~mm}$ do CCN. Neste intervalo o VE aumentou 67 vezes, enquanto o CCN aumentou apenas 4,4 vezes. CONCLUSÕES: o VE é um parâmetro mais sensível que o CCN para avaliar o crescimento embrionário entre a sétima e a décima semana de gestação.

\section{Abstract}

PURPOSE: to evaluate the embryo's volume (EV) between the seventh and the tenth gestational week, through tridimensional ultrasonography. METHODS: a transversal study with 63 normal pregnant women between the seventh and the tenth gestational week. The ultrasonographical exams have been performed with a volumetric abdominal transducer. Virtual Organ Computer-aided Analysis (VOCAL) has been used to calculate EV, with a rotation angle of $12^{\circ}$ and a delimitation of 15 sequential slides. The average, median, standard deviation and maximum and minimum values have been calculated for the EV in all the gestational ages. A dispersion graphic has been drawn to assess the correlation between EV and the craniogluteal length (CGL), the adjustment being done by the determination coefficient $\left(R^{2}\right)$. To determine EV's reference intervals as a function of the $C G L$, the following formula was used: percentile=EV+K versus SD, with $K=1.96$. RESULTS: CGL has varied from 9.0 to $39.7 \mathrm{~mm}$, with an average of $23.9 \mathrm{~mm}( \pm 7.9 \mathrm{~mm})$, while EV has varied from 0.1 to $7.6 \mathrm{~cm}^{3}$, with an average of $2.7 \mathrm{~cm}^{3}\left( \pm 3.2 \mathrm{~cm}^{3}\right)$. EV was highly correlated to $C G L$, the best adjustment being obtained with quadratic regression ( $E V=0.2-0.055$ versus $C G L+0.005$ versus $\left.C G^{2} ; R^{2}=0.8\right)$. The average EV has varied from $0.1\left(-0.3\right.$ to $\left.0.5 \mathrm{~cm}^{3}\right)$ to $6.7 \mathrm{~cm}^{3}\left(3.8\right.$ to $\left.9.7 \mathrm{~cm}^{3}\right)$ within the interval of 9 to $40 \mathrm{~mm}$ of CGL. EV has increased 67 times in this interval, while CGL, only 4.4 times. CONCLUSIONS: EV is a more sensitive parameter than CGL to evaluate embryo growth between the seventh and the tenth week of gestation.
Setor de Ultra-sonografia Tridimensional do Departamento de Obstetrícia da Universidade Federal de São Paulo - UNIFESP - São Paulo (SP), Brasil.

'Pós-graduando do Departamento de Obstetrícia da Universidade Federal de São Paulo - UNIFESP - São Paulo (SP), Brasil.

2 Professor Adjunto e Chefe da Disciplina de Medicina Fetal do Departamento de Obstetrícia da Universidade Federal de São Paulo - UNIFESP - São Paulo (SP), Brasil.

${ }_{3}^{3}$ Professor Afiliado do Departamento de Obstetrícia da Universidade Federal de São Paulo - UNIFESP - São Paulo (SP), Brasil.

${ }^{4}$ Mestre pelo Departamento de Obstetrícia da Universidade Federal de São Paulo - UNIFESP - São Paulo (SP), Brasil.

${ }_{5}^{5}$ Professor Titular do Departamento de Obstetrícia da Universidade Federal de São Paulo - UNIFESP - São Paulo (SP), Brasil. 


\section{Introdução}

O período embrionário é crítico para o desenvolvimento humano, pois todos os sistemas orgânicos são formados nesta fase ${ }^{1}$. O entendimento do desenvolvimento normal da gestação inicial e a observação deste processo permitem-nos monitorar efetivamente a gestação de primeiro trimestre e reconhecer sinais precoces de gestações anormais ${ }^{2}$. Tradicionalmente, a medida do comprimento cabeça-nádega $(\mathrm{CCN})$ por meio da ultra-sonografia bidimensional tem sido o método mais acurado para determinação da idade gestacional ${ }^{3} \mathrm{e}$, em alguns casos para o diagnóstico precoce de restrição do crescimento em fetos com anomalias cromossômicas ${ }^{4}$.

O advento da ultra-sonografia tridimensional (US3D) possibilitou uma aferição volumétrica mais acurada de objetos com formatos irregulares ${ }^{5}$. Em relação à aferição do volume do embrião (VE), os estudos publicados utilizaram tanto o software EchoPac $3 \mathrm{D}^{6}$ quanto o VOCAL (Virtual Organ Computer-aided Analysis) ${ }^{7-9}$. O método VOCAL apresenta como vantagem a possibilidade de modificação do contorno em cada plano, o que permite que pequenos ajustes sejam realizados, de forma a tornar a aferição volumétrica mais acurada ${ }^{10}$.

A determinação do VE parece ser um método mais preciso do que o $\mathrm{CCN}$, para a detecção precoce de restrição do crescimento no primeiro trimestre de gestação. Em recente estudo, o volume fetal entre a $7^{\mathrm{a}}$ e a $12^{\mathrm{a}}$ semana de gestação aumentou 35 vezes, ao contrário do $\mathrm{CCN}$, que aumentou apenas 4,5 vezes $^{7}$. A mensuração do volume da cabeça e do tronco se mostrou um método mais acurado que o $\mathrm{CCN}$ para a detecção precoce de restrição do crescimento em fetos com cromossomopatias ${ }^{11}$.

O objetivo deste estudo é avaliar a evolução do VE entre a sétima e a décima semanas de gestação, como base para futuros estudos em gestações com risco aumentado para restrição precoce do crescimento.

\section{Métodos}

Realizou-se um estudo do tipo corte transversal, entre novembro de 2005 e março de 2007, com 63 gestantes entre a sétima e a décima semana de gestação. Este estudo foi aprovado pelo Comitê de Ética em Pesquisa da Universidade Federal de São Paulo (UNIFESP-EPM) sob o n. 1492/06, sendo que as pacientes que concordaram com a participação voluntária assinaram o termo de consentimento.

Todas as pacientes foram selecionadas no setor de prénatal de baixo risco do Departamento de Obstetrícia da UNIFESP-EPM, sendo estas oriundas do Sistema Único de Saúde do município e da região metropolitana de São Paulo. Os critérios de inclusão foram os seguintes: gestação única com embrião vivo e idade gestacional determinada pela data da última menstruação e confirmada por ultra-sonografia realizada até a décima semana utilizando como parâmetro o CCN. Os critérios de exclusão foram: gestantes portadoras de doenças crônicas (diabetes mellitus, hipertensão arterial e colagenoses), tabagistas e usuárias de drogas ilícitas na atual gestação e sangramento vaginal na atual gestação.

Todos os exames foram realizados no setor de US3D do Departamento de Obstetrícia da UNIFESP-EPM, sendo estes realizados por apenas dois examinadores com experiência em US3D em Obstetrícia. As análises off-line para a mensuração do VE foram realizadas por apenas um examinador utilizando o software SonoView Pro versão 1.03 (Medison, Seoul, Korea). Todas as pacientes foram avaliadas uma única vez (corte transversal), sendo os exames realizados em dois aparelhos da marca Medison (SA-8000LIVE e Accuvix XQ) equipados com transdutores endocavitários volumétricos e multifreqüenciais.

Inicialmente, realizou-se uma avaliação bidimensional em tempo real de forma a se medir o $\mathrm{CCN}$, o diâmetro médio do saco gestacional (média aritmética dos três maiores diâmetros) e avaliar a freqüência cardíaca embrionária. Em seguida, ativou-se a tecla 3D, aparecendo a janela (BOX) tridimensional. Utilizou-se um ângulo de abertura de $30^{\circ}$ e velocidade de varredura normal, diminuindo os artefatos de transmissão em decorrência da movimentação embrionária. O BOX foi posicionado de forma a englobar apenas o embrião (ROI - região de interesse). Após a varredura tridimensional, a imagem foi apresentada na tela na forma de três planos ortogonais: axial, sagital e coronal. Selecionou-se como referencial o plano sagital, sendo este magnificado em $50 \%$. Em seguida, a imagem do embrião foi deslocada para o centro do plano e rodada em torno do eixo "y" de forma que o embrião se dispusesse verticalmente. Em seqüência, ativou-se a tecla VOCAL com ângulo de $12^{\circ}$ e modo de delimitação manual dos planos. Os calibradores de medida foram posicionados nos pólos do embrião e após a delimitação de 15 planos seqüenciais, o aparelho fornecia a imagem reconstruída da estrutura com o seu volume em $\mathrm{cm}^{3}$.

Os dados foram armazenados em planilha do programa Excel (Microsoft, USA) e analisados por meio de um programa estatístico SPSS for Windows versão 13.0 (SPSS Inc., Chicago, IL, USA). Para o CCN e o VE foram calculadas médias, medianas, desvios padrão $(\mathrm{dp})$ e valores máximo e mínimo para a idade materna. Para se avaliar a correlação entre o VE e o CCN, foi criado diagrama de dispersão, sendo o ajuste da equação determinado pelo coeficiente de determinação $\left(\mathrm{R}^{2}\right)$. Para a determinação de intervalos de referência do VE em função do CCN, utilizou-se o método proposto por Royston e Wright ${ }^{12}$. Este método consiste em se ajustar um modelo de regressão para as medidas do VE considerando o $\mathrm{CCN}$ como variável independente. Em seguida, ajusta-se um modelo para os 
dp segundo o $\mathrm{CCN}$, considerando-se como resposta os resíduos absolutos do modelo anterior. A partir do VE e dp ajustados para cada valor de CCN, calculam-se os percentis de acordo com a fórmula: percentil $=\mathrm{VE}+\mathrm{K}$ versus dp. Para se obterem os intervalos de referência de $95 \%$, calculam-se os percentis 2,5 e 97,5 em que o valor de $K=1,96$. Em todas as análises, utilizou-se nível de significância (p) de 0,05.

\section{Resultados}

Foram selecionadas 63 gestantes entre a sétima e a décima semana, sendo que todas preencheram os critérios de inclusão, não sendo excluídas gestantes que inicialmente foram incluídas. A idade materna variou de 20 a 41 anos, com média de 30,1 anos ( $\pm 5,8$ anos). O número de gestações variou de um a nove, com média de 2,3 gestações $( \pm 1,7$ gestação). A idade gestacional variou de sete a dez semanas, com média de 8,7 semanas ( \pm uma semana). O CCN variou de 9,0 a $39,7 \mathrm{~mm}$, com média de $23,9 \mathrm{~mm}$ $( \pm 7,9 \mathrm{~mm})$. O VE variou de 0,1 a $7,6 \mathrm{~cm}^{3}$, com média $2,70 \mathrm{~cm}^{3}( \pm 1,7)$. O VE médio variou de $0,4\left( \pm 0,3 \mathrm{~cm}^{3}\right)$ a $4,9 \mathrm{~cm}^{3}\left( \pm 1,5 \mathrm{~cm}^{3}\right)$ entre a sétima e a décima semana. A Tabela 1 mostra a média, mediana, dp e valores máximo e mínimo do VE em cada idade gestacional avaliada. O VE mostrou-se altamente correlacionado com o CCN, sendo que o melhor ajuste foi uma regressão quadrática $\left(R^{2}=0,853\right)$. A equação que melhor expressa esta correlação é a seguinte:

Tabela 1 - Médias, medianas, desvios padrão e valores máximos e mínimos para o volume do embrião entre a sétima e a décima semana de gestação

\begin{tabular}{lccccccc}
\hline & IG & n & Média & dp & Mediana & Mínimo & Máximo \\
\hline Volume & 7 & 10 & 0,4 & 0,3 & 0,3 & 0,1 & 1,1 \\
do embrião & 8 & 17 & 1,1 & 0,5 & 1,0 & 0,1 & 2,1 \\
$\left(\mathrm{~cm}^{2}\right)$ & 9 & 22 & 2,3 & 1,0 & 1,9 & 1,3 & 4,5 \\
& 10 & 14 & 4,9 & 1,5 & 4,9 & 2,3 & 7,6 \\
\hline
\end{tabular}

IG=idade gestacional; $n=$ número de gestantes em cada idade gestacional; $\mathrm{dp}=$ desvio padrão.

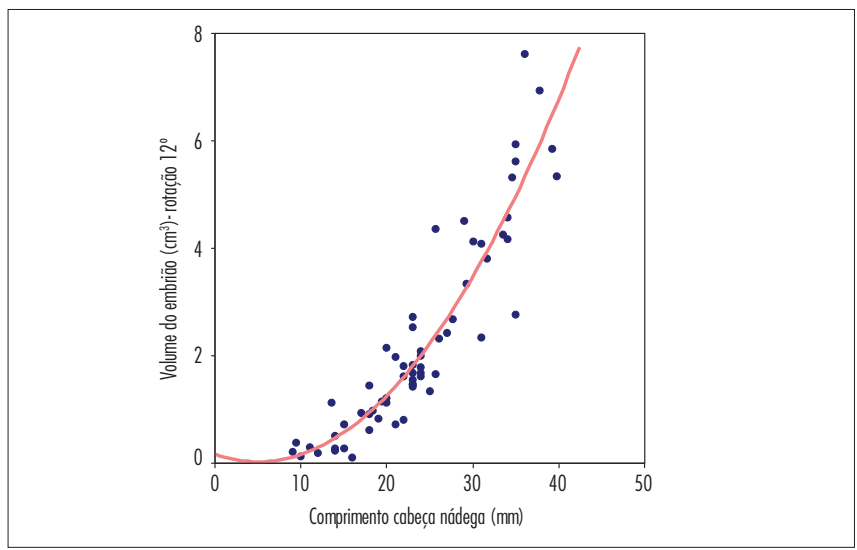

Figura 1 - Gráfico de dispersão do volume do embrião em função do comprimento cabeça-nádega, com o ajuste da regressão quadrática (linha vermelha).
$\mathrm{VE}=0,2-0,055$ versus $\mathrm{CCN}+0,005$ versus $\mathrm{CCN}^{2}$. A Figura 1 mostra o gráfico de dispersão do VE em função do CCN. Observa-se a forte correlação entre ambos, com a maioria dos pontos próximos à diagonal do gráfico.

A Figura 2 mostra o gráfico de ajuste da regressão quadrática incluindo os valores de referência do VE entre os percentis 2,5 e 97,5. Observou-se nesta figura que a maioria dos pontos se dispuseram em torno da média. A Tabela 2 mostra os valores médios esperados, com os respectivos limites inferior e superior do VE entre 9 e $40 \mathrm{~mm}$ de CCN. Observou-se que a média do VE variou de 0,1 $\left(-0,3\right.$ a $\left.0,5 \mathrm{~cm}^{3}\right)$ a $6,7 \mathrm{~cm}^{3}\left(3,8\right.$ a $\left.9,7 \mathrm{~cm}^{3}\right)$ nesse intervalo do CCN, ou seja, houve um aumento de 67 vezes do VE, enquanto que o $\mathrm{CCN}$ aumentou apenas 4,4 vezes.

Tabela 2 - Volumes médios do embrião com os respectivos limites inferior e superior em função do comprimento cabeça-nádega

\begin{tabular}{|c|c|c|c|}
\hline \multirow{2}{*}{ CCN (mm) } & \multirow{2}{*}{$\begin{array}{l}\text { Volume esperado } \\
\text { do embrião }\left(\mathrm{cm}^{3}\right)\end{array}$} & \multicolumn{2}{|c|}{ Intervalo de referência } \\
\hline & & Limiłe inferior & Limite superior \\
\hline 9 & 0,1 & $-0,3$ & 0,5 \\
\hline 10 & 0,2 & $-0,3$ & 0,6 \\
\hline 11 & 0,2 & $-0,2$ & 0,7 \\
\hline 12 & 0,3 & $-0,2$ & 0,7 \\
\hline 13 & 0,4 & $-0,1$ & 0,9 \\
\hline 14 & 0,5 & 0 & 1,0 \\
\hline 15 & 0,6 & 0 & 1,1 \\
\hline 16 & 0,7 & 0,1 & 1,3 \\
\hline 17 & 0,8 & 0,2 & 1,4 \\
\hline 18 & 0,9 & 0,3 & 1,6 \\
\hline 19 & 1,1 & 0,3 & 1,8 \\
\hline 20 & 1,3 & 0,4 & 2,1 \\
\hline 21 & 1,4 & 0,5 & 2,3 \\
\hline 22 & 1,6 & 0,7 & 2,5 \\
\hline 23 & 1,8 & 0,8 & 2,8 \\
\hline 24 & 2,0 & 0,9 & 3,1 \\
\hline 25 & 2,2 & 1,0 & 3,4 \\
\hline 26 & 2,4 & 1,2 & 3,7 \\
\hline 27 & 2,7 & 1,3 & 4,0 \\
\hline 28 & 2,9 & 1,5 & 4,4 \\
\hline 29 & 3,2 & 1,6 & 4,7 \\
\hline 30 & 3,4 & 1,8 & 5,1 \\
\hline 31 & 3,7 & 2,0 & 5,5 \\
\hline 32 & 4,0 & 2,1 & 5,9 \\
\hline 33 & 4,3 & 2,3 & 6,3 \\
\hline 34 & 4,6 & 2,5 & 6,8 \\
\hline 35 & 5,0 & 2,7 & 7,2 \\
\hline 36 & 5,3 & 2,9 & 7,7 \\
\hline 37 & 5,6 & 3,1 & 8,2 \\
\hline 38 & 6,0 & 3,3 & 8,7 \\
\hline 39 & 6,4 & 3,5 & 9,2 \\
\hline 40 & 6,7 & 3,8 & 9,7 \\
\hline
\end{tabular}

$\mathrm{CCN}=$ comprimento cabeça-nádega. 


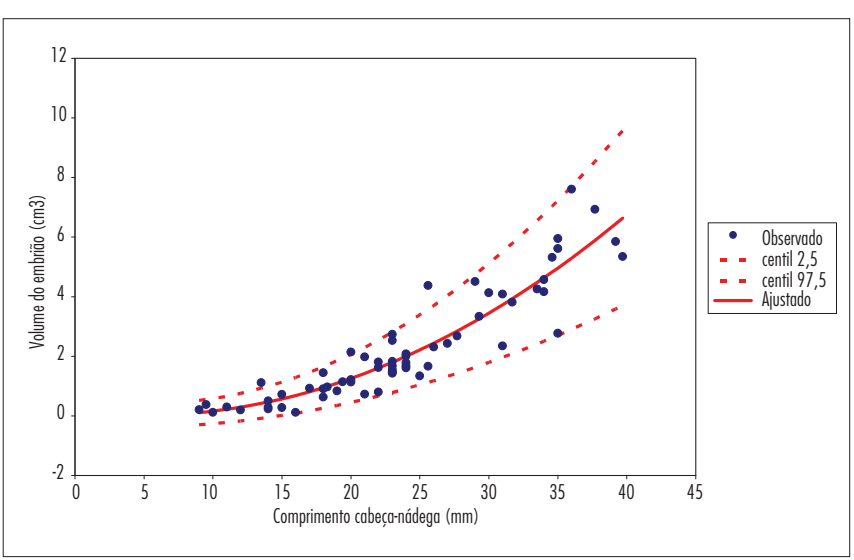

Figura 2 - Gráfico da regressão quadrática incluindo os intervalos de referência 2,5 e 97,5 entre 9 e 45 mm do comprimento cabeça-nádega.

\section{Discussão}

$\mathrm{O}$ advento dos transdutores endocavitários de alta freqüência permitiu a obtenção de imagens detalhadas das estruturas embrionárias ${ }^{13}$. $\mathrm{O}$ advento da US3D permitiu uma melhor avaliação das estruturas superficiais de embriões e fetos jovens nos modos de renderização ${ }^{14} \mathrm{e}$ multiplanar ${ }^{15}$. Os transdutores volumétricos endocavitários permitem uma avaliação em superfície pormenorizada do desenvolvimento do embrião ${ }^{16}$.

A primeira descrição de reconstrução tridimensional de embriões humanos foi publicada no final da década passada ${ }^{17}$. Utilizou-se um transdutor endocavitário volumétrico de $7,5 \mathrm{MHz}$ para a aquisição dos volumes, sendo o processamento realizado com o software EchoPAC-3D. Para a reconstrução tridimensional das estruturas embrionárias, as imagens foram segmentadas pela delimitação manual de múltiplos planos bidimensionais paralelos. A partir destes contornos, poliedros foram criados para definir a superfície e o volume das estruturas. Concluiu-se que aquele sistema é capaz de reconstruir estruturas com menos de $10 \mathrm{~mm}$, permitindo monitorizar o desenvolvimento embrionário.

Neste estudo, optamos pelo uso do software VOCAL que, diferentemente do EchoPAC-3D, está disponível em diversos aparelhos de US3D. Os métodos VOCAL e multiplanar se mostraram concordantes na avaliação volumétrica de objetos com medidas e formas diferentes ${ }^{18}$; além do mais, recentemente nosso grupo comprovou que ambas as técnicas, VOCAL com rotação de 12 e $30^{\circ}$ e multiplanar com intervalo de $1,0 \mathrm{~mm}$, se mostraram concordantes quanto à medida volumétrica da placenta entre a sétima e a décima semana ${ }^{19}$. Além disso, o método VOCAL mostrou-se uma técnica reprodutível para a avaliação do volume do tronco e da cabeça fetal entre a $11^{a}$ semana e 13 semanas e seis dias ${ }^{8}$. A opção pela técnica VOCAL se deveu ao fato de ser uma técnica mais rápida e menos dependente do operador que a multiplanar e, com condições de ser aplicada à prática clínica. Em relação ao ângulo de rotação, optamos pela rotação em $12^{\circ} \mathrm{com}$ delimitação de 15 planos consecutivos, apesar de a maioria das publicações utilizar rotação em $30^{\circ} \mathrm{com}$ delimitação de seis planos ${ }^{7,8,11}$. O software presente em nossos aparelhos de US3D (SonoView Pro) permite a rotação pelo método VOCAL em apenas 12,18 e $30^{\circ}$. A opção por $12^{\circ}$ se deveu ao maior número de planos a serem delimitados, de forma a aumentar a acurácia da medida. Na avaliação do volume do endométrio ${ }^{20}$, os volumes médios obtidos com rotação em $30^{\circ}$ e pelo método multiplanar foram significativamente menores que os obtidos pelas rotações em 6 e $15^{\circ}$. Recentemente, o método VOCAL com rotação em $15^{\circ}$, com delimitação de 12 planos consecutivos, foi utilizado para a determinação volumétrica da cabeça e do tronco de fetos únicos e gemelares provenientes de fertilização in vitro ${ }^{9}$.

Neste estudo, foi incluída a medida do volume dos membros juntamente com a cabeça e o tronco, pois após a décima semana torna-se muito difícil a delimitação adequada dos membros fetais. Além do mais, o método VOCAL permite o cálculo de apenas um volume por vez, tornando desnecessária a segmentação do corpo. Em estudo realizado com o software EchoPac3D, os autores segmentaram o corpo fetal (cabeça, tronco e membros) de 44 fetos entre 7 e 12 semanas e observaram que entre a sétima e a décima semana o volume dos membros aumentou apenas de 5 a $8 \%$ como proporção do corpo inteiro ${ }^{6}$. Em estudos realizados entre a $11^{\text {a }}$ semana e 13 semanas e seis dias, os autores avaliaram apenas o volume da cabeça e do tronco fetais pelo método VOCAL, pois neste período gestacional os membros estão cruzados e as mãos freqüentemente estão em contato com a face, o que poderia tornar a medida volumétrica menos reprodutível ${ }^{8,11}$.

Neste estudo, observamos uma forte correlação entre o VE e o CCN, sendo esta do tipo exponencial. Esta mesma correlação foi obtida por Aviram et al. ${ }^{7}$, os quais utilizaram o método VOCAL com rotação em $30^{\circ}$, e por Blaas et al. ${ }^{6}$, que utilizaram o EchoPac3D. Recentemente, comprovou-se também correlação exponencial entre os volumes da cabeça e do tronco fetais, obtidos pelo método VOCAL com rotação em $15^{\circ}$ e, o CCN tanto para fetos únicos quanto gemelares concebidos por fertilização in vitro ${ }^{9}$.

$\mathrm{Na}$ comparação das médias do VE, observou-se que os valores obtidos neste estudo $\left(0,4 \mathrm{~cm}^{3}\right.$ na sétima semana e $4,9 \mathrm{~cm}^{3}$ na décima semana) se aproximaram muito de outro estudo que utilizou a mesma técnica volumétrica (0,4 $\mathrm{mL}$ na sétima semana e 4,6 $\mathrm{mL}$ na décima semana). Entretanto, em comparação ao estudo que utilizou o software EchoPac3D, as medidas foram superestimadas ${ }^{6}$. A explicação para isso pode ser que no presente estudo foi realizada a mensuração do volume dos membros juntamente 
com o do corpo, pois o método VOCAL não permite o cálculo volumétrico preciso por segmentos (cabeça, tronco e membros), diferentemente do EchoPac3D.

Neste estudo, optou-se por determinar intervalos de referência para o VE em função do CCN, pois este é o principal parâmetro para se avaliar a idade gestacional e o crescimento embrionário no primeiro trimestre. Esta correlação foi avaliada em todos os estudos sobre volumetria do embrião/fetos jovens, de forma a pesquisar se o VE é um parâmetro mais sensível que o CCN para o diagnóstico de distúrbios precoces do crescimento no primeiro trimestre ${ }^{6-9}$.

Neste estudo, o CCN variou de 9,0 a $40 \mathrm{~mm}$ (aumento de 4,4 vezes), enquanto que no mesmo período o $\mathrm{VE}$ variou de 0,1 a $6,7 \mathrm{~cm}^{3}$ (aumento de 67 vezes), comprovando ser o VE um parâmetro mais sensível que o CCN para o diagnóstico precoce de distúrbios do crescimento no primeiro trimestre de gestação. Em estudo realizado por Aviram et al. ${ }^{7}$, no período entre a $7^{\mathrm{a}}$ e a $12^{\mathrm{a}}$ semanas, enquanto o $\mathrm{CCN}$ aumentou 4,5 vezes o $\mathrm{VE}$ aumentou 35 vezes. Em estudo realizado em fetos com 11 a 13 semanas e seis dias, com a mesma técnica utilizada no presente estudo, o volume da cabeça e do tronco aumentou cinco a seis vezes, enquanto o CCN apenas dobrou o seu valor ${ }^{8}$. Tal resultado mostra que o VE é um parâmetro promissor na avaliação do desenvolvimento do embrião no primeiro trimestre, podendo futuramente ser aplicado na prática clínica em gestações com risco aumentado de resultados adversos, como a restrição do crescimento intra-uterino ou cromossomopatias.

A maior sensibilidade do VE em relação ao CCN para avaliar o crescimento embrionário determinada neste estudo necessita ser comprovada em estudos futuros com maiores casuísticas.
1. Harkness LM, Rodger M, Baird DT. Morphological and molecular characteristics of living human fetuses between Carnegie stages 7 and 23: ultrasound scanning and direct measurements. Hum Reprod Update. 1997;3(1):25-33.

2. Jurkovic D, Gruboeck K, Campbell S. Ultrasound features of normal early pregnancy development. Curr Opin Obstet Gynecol. 1995;7(6):493-504.

3. Hadlock FP, Shah YP, Kanon DJ, Lindsey JV. Fetal crown-rump length: reevaluation of relation to menstrual age (5-18 weeks) with high-resolution real-time US. Radiology. 1992;182(2):501-5.

4. Kuhn P, Brizot ML, Pandya PP, Snijders RJ, Nicolaides KH. Crownrump length in chromosomally abnormal fetuses at 10 to 13 weeks' gestation. Am J Obstet Gynecol. 1995; 172(1 Pt 1):32-5.

5. Riccabona $M$, Nelson TR, Pretorius $D H$. Three-dimensional ultrasound: accuracy of distance and volume measurements. Ultrasound Obstet Gynecol. 1996;7(6):429-34.

6. Blaas HG, Taipale P, Torp H, Eik-Nes SH. Three-dimensional ultrasound volume calculations of human embryos and young fetuses: a study on the volumetry of compound structures and its reproducibility. Ultrasound Obstet Gynecol. 2006;27(6):640-6.

7. Aviram R, Shpan DK, Markovitch O, Fishman A, Tepper R. Threedimensional first trimester fetal volumetry: comparison with crown rump length. Early Hum Dev. 2004;80(1):1-5.

8. Falcon $\mathrm{O}$, Peralta $\mathrm{CF}$, Cavoretto P, Faiola $\mathrm{S}$, Nicolaides $\mathrm{KH}$. Fetal trunk and head volume measured by three-dimensional ultrasound at $11+0$ to $13+6$ weeks of gestation in chromosomally normal pregnancies. Ultrasound Obstet Gynecol. 2005;26(3):263-6.

9. Martins WP, Ferriani RA, Nastri CO, Filho FM. First trimester fetal volume and crown-rump length: comparison between singletons and twins conceived by in vitro fertilization. Ultrasound Med Biol. 2008;34(9): 1360-4.

10. Peralta CF, Cavoretto P, Csapo B, Falcon O, Nicolaides KH. Lung and heart volumes by three-dimensional ultrasound in normal fetuses at 12-32 weeks' gestation. Ultrasound Obstet Gynecol. 2006;27(2):128-33
11. Falcon O, Peralta CF, Cavoretto P, Auer M, Nicolaides KH. Fetal trunk and head volume in chromosomally abnormal fetuses at $11+0$ to $13+6$ weeks of gestation. Ultrasound Obstet Gynecol. $2005 ; 26(5): 517-20$.

12. Royston $P$, Wright EM. How to construct 'normal ranges' for fetal variables. Ultrasound Obstet Gynecol. 1998; 11 (1):30-8.

13. Timor-Tritsch IE, Farine D, Rosen MG. A close look at early embryonic development with the high-frequency transvaginal transducer. Am J Obstet Gynecol. 1988;159(3):676-81.

14. Hata T, Aoki S, Manabe A, Hata K, Miyazaki K. Three-dimensional ultrasonography in the first trimester of human pregnancy. Hum Reprod. 1997;12(8):1800-4.

15. Hull AD, James G, Salerno CC, Nelson T, Pretorius DH. Threedimensional ultrasonography and assessment of the first-trimester fetus. J Ultrasound Med. $2001 ; 20(4): 287-93$.

16. Zanforlin Filho SM, Araujo Júnior E, Guimarães Filho HA, Pires CR, Nardozza LM, Moron AF. Sonoembryology by threedimensional ultrasonography: pictorial essay. Arch Gynecol Obstet. 2007;276(2):197-200.

17. Blaas HG, Eik-Nes SH, Berg S, Torp H. In-vivo three-dimensional ultrasound reconstructions of embryos and early fetuses. Lancet. 1998;352(9135): 1 182-6.

18. Raine-Fenning NJ, Clewes JS, Kendall NR, Bunkheila AK, Campbell BK, Johnson IR. The interobserver reliability and validity of volume calculation from three-dimensional ultrasound datasets in the in vitro setting. Ultrasound Obstet Gynecol. 2003;21(3):283-91.

19. Nowak PM, Nardozza LM, Araujo Júnior E, Rolo LC, Moron AF. Comparison of placental volume in early pregnancy using multiplanar and VOCAL methods. Placenta. 2008;29(3):241-5.

20. Raine-Fenning N, Campbell B, Collier J, Brincat M, Johnson I. The reproducibility of endometrial volume acquisition and measurement with the VOCAL-imaging program. Ultrasound Obstet Gynecol. 2002; 19(1):69-75. 